\title{
Numerical grading of clinical neurological status after serious head injury
}

\author{
J OH N K. YEN, ROBERT S. B OURKE, LOUIS R. NELSON,
} A NDA. JOH N POPP

From the Division of Neurosurgery, Albany Medical College, Albany, New York, USA

SUMMARY A scheme to quantitate the clinical neurological status of the seriously headinjured patient has been devised. The neurological parameters used to quantify the degree of injury are based on neurological functions which have previously been accepted as indicators of the severity of the head injury. A numerical value is assigned to each parameter with emphasis on defining the level of consciousness. The accrued point total of each examination represents the neurological status of the patient at that time. Mean values and standard error from the means are determined from repeated examinations during a single 24 hour period, and are plotted against days after injury. From this graph a line which represents the rate of clinical recovery is determined by least squares analysis. General intensive care nurses were trained to score patients independently; their determinations were found to be in statistical agreement with scores derived from examinations by the attending physicians. The data presented highlight the effects of hypoxaemia in impeding the rate of neurological recovery from a serious head injury. This simple clinical analytical scheme for the quantitative assessment of patients with head injury permits evaluation of the efficacy of various modes of therapy in altering the rate of recovery.

Methods have been proposed for the numerical assessment of the neurological status in specific disorders of the central nervous system (Kurtzke, 1955; Tourtellote et al., 1965; Mathew and Lawson, 1966; Lovejoy et al., 1974). Previous methods of assessment of the head-injured patient have focused on parameters which may predict survival and recovery (Adams and McComb, 1953; Carlsson et al., 1968; Overgaard et al., 1973; Teasdale and Jennett, 1974; Jennett and Bond, 1975; Jennett, 1976). Only one scale (Jennett and Teasdale, 1977) is currently available which provides a numerical assessment of the severity of head injury together with a methodology to quantitate the rate of restitution of neurological function of survivors. However, this assessment is limited in specific and commonly seen clinical situations.

A scalar approach to the clinical assessment of the neurological status is necessary to compare accurately the results of the various regimes used in the care of the seriously head-injured. It was our intention to provide a reproducible and statistically

\footnotetext{
Address for reprint requests: Dr R. S. Bourke, Division of Neurosurgery, Albany Medical College, Albany, NY 12208, USA. Accepted 11 May 1978
}

reliable rating method based on parameters of the clinical neurological examination previously determined by others to be valid in the assessment of the seriously head-injured. The clinical rating scheme was designed to be succinct and readily applied either by physicians or general intensive care nursing personnel with no special neurosurgical nursing expertise. Furthermore, the scale was designed to be useful from initial evaluation of the intubated head-injured patient until achievement of personal self-care.

\section{Description}

The general parameters of the Head Injury Watch Sheet (HIWS) (Table 1) selected to reflect the neurological status of the seriously head-injured were modified from criteria already shown to be valid for assessing the head-injured (Bouzarth, 1968; Teasdale and Jennett, 1974). These criteria were divided into three major categories: $(A)$ consciousness and mental status; $(B)$ motor response to pain; $(C)$ pupillary reaction. These categories were further divided into six subcategories composed of a series of testable parameters together 
Table 1 Head Injury Watch Sheet $(H I W S)$

(A) Tests of consciousness and mental status

I Response to verbal command

Unit points

(a) Complex-responds to intricate verbal commands (feed oneself, button and unbutton clothing)

(b) Immediate, simple-responds to commands promptly and appropriately (squeeze hands, blink eyes)

(c) Delayed, simple-response to commands is delayed and requires reinforcement (repeated several times)

(d) Questionable-response to simple command is inappropriate or doubtful

(e) No response-no response to simple verbal commands

II Stimulus to awaken

(a) Alert-patient is awake and immediately responsive to his name

(b) Shaking - requires gentle shaking to awaken patient

(c) Light pain-requires mild painful stimulus to awaken patient (pinprick)

(d) Deep pain-requires deep sternal pressure or squeeze of trapezius to awaken patient

(e) None-will not awaken to any stimulus or patient may open eyes but no voice contact can be made (no response to verbal command)

III Quality of consciousness

(a) Oriented-patient appreciates place, person, and date

(b) Confused-patient is uncertain of personal situation, month, place

(c) Restless-patient is agitated and occasional contact by voice can be made (patient will attend and respond to command)

(d) Combative-patient is restless, difficult to control and meaningful contact by voice cannot be made (patient will not attend or respond to commands)

(e) None-no contact by voice can be made

B) Motor response to pain

IV Non-verbal reaction to pain

(a) Appropriate-reacts immediately to any painful stimulus (withdrawing hand or foot to pinching or shaking head to squeezing of trapezius)

(b) Delayed - there is a pause or slowness in appropriate response to painful stimulus (includes attempts at disconnecting IV lines, respiratory apparatuses etc). No evidence of decortication or decerebration

(c) Inappropriate - evidence of decortication and/or occasional unilateral decerebration

(d) Decerebrate-bilateral decerebration

(e) None-no responses

V Ability to move (each extremity)

(a) Brisk-rapid and forceful movement of limb to painful stimulus

(b) Slow-any delay or weakness in movement of limb to painful stimulus

(c) Absent-response to pain may be absent or decerebrate/decorticate

4

1

0

4

(C) Pupils

VI Reaction

(a) Brisk-rapid constriction to direct light

(b) Slow-a delay and/or partial constriction to direct light

(c) Questionable-cannot be sure if pupil is constricting to direct light

(d) No reaction to light with their corresponding definitions and relative numerical value. The necessity for frequent repeated examinations by the nursing staff required a succinct scalar system, the completion of which would not become burdensome. A more thorough clinical neurological examination (Denny-Brown, 1950), including evaluation of brainstem function (Plum and Posner, 1972), was carried out periodically by the neurosurgical staff.

A maximum score of 30 points is accrued by adding the numerical value assigned to each category constituting the examination. Twelve of the 30 points are allocated to the evaluation of conciousness and mental status, 12 points reflect motor activity and response to pain, and six of the 30 points reflect grading of pupillary reaction. Point totals for each category are determined by the sum of the best response in each subcategory at that point in time except in subcategories IV and $V$, where the score reflects the worst response-that is, decerebration or decortication-even if it is transient. Achievement of a maximum score by any patient is meant to describe a verbally appropriate patient who could provide the rudiments of self-care such as buttoning and unbuttoning clothing, feeding, and management of the essentials of elimination.

Patients were evaluated neurologically and scored hourly by intensive care nurses. The HIWS of each patient was analysed daily by determining a mean value ( $\pm S E$ ) for each of the three nursing shifts together with the total 24 hour period. Comparable scoring was carried out independently by physicians. Point totals were then plotted as a function of time, and the slopes were determined by the method of least squares. The slope represents the rate of neurological recovery expressed in points per day.

Several brief sessions were necessary to instruct general intensive care nurses in the use of the HIWS properly. Specific test examples together with readily understandable and applicable defi- 
nitions facilitated the use and acceptance by the nurses. It was important that the nurses clearly appreciated decerebrate and decorticate posturing since the category motor response to pain (HIWS category B, subcategory IV) was based on the presence of these pathological postures. When the response to a painful stimulus was other than decerebration or decortication, the best motor response of each extremity was noted and grading was carried out in both subcategories IV and V.

A source of confusion occurred in distinguishing the conscious patient from the vegetative (Jennett and Plum, 1972) patient who displayed sleep-wake cycles. This was resolved by indicating to the nurses the ability of the conscious patient to follow a verbal command-for example, squeeze hands and blink eyes. Nurses were instructed to command the patient to release his grip, a positive response to the command indicating volitional action and consciousness.

The following, in outline form, explains the components of the watch sheet.

(A) Tests of consciousness and mental status are composed of three subcategories: (I) response to verbal command, (II) stimulus to awaken, and (III) quality of consciousness.

Response to verbal command is represented by graded responses to various specific instructions given to the patient - that is, feed yourself, squeeze hands. Were the patient to have an arm in a cast, a clear attempt to comply with the command would be accepted.

Stimulus to awaken Various stimuli of increasing intensity (pinprick, deep sternal pressure) required to awaken the patient are given as examples to be used by the nurses. This helps in assessing the patient's level of consciousness.

Quality of consciousness refers to the patient's thought content or mental status, and ranges from a confused, agitated state to a fully oriented one.

(B) Tests of motor response to pain are divided into the following subcategories: (IV) non-verbal reaction to pain, and (V) ability to move.

Non-verbal reaction to pain refers to the generalised motor response that occurs spontaneously or as a result of an external stimulus. In patients that display decerebrate or decorticate posturing, however transient, scores of one to two points are recorded until the patient entirely ceases exhibiting these pathological motor movements.

Ability to move Individual extremities are evaluated for paresis in the absence of abnormal posturing. It was necessary to instruct the nursing staff that the vigorous maintenance of decerebrate or decorticate posturing should not be interpreted as normal "brisk" movement and given two points on the scale. The presence of decerebrate or decorticate response whether unilateral or bilateral results in a score of zero points in all extremities in this category.

(C) Reaction of pupils has only one category (VI) in which graded responses of each pupil to direct light are determined. A pupillary gauge is used to estimate the size of each pupil, and this measurement is recorded.

\section{Illustrative cases}

\section{CASE 1}

A 7 year old boy (PO) was involved in a car accident and sustained a severe head injury. He was intubated, given supplemental oxygen, and cerebral angiograms were obtained and interpreted as normal. On admission, he was unilaterally decerebrate and his right pupil was dilated and unreactive to light. He received two points for being unilaterally decerebrate from subcategory IV, nonverbal reaction to pain, and three points for having a briskly reactive left pupil, subcategory VI, reaction of pupils. His scores in the other subcategories (I, II, III, V) were zero. His total admission score was five points. Decerebrate responses ceased on the following day, and he gradually improved at a rate of 2.34 points/day. On day five, he responded briskly and appropriately to painful stimuli in three limbs, but the right arm was noted to be weak. The right pupil continued to react briskly with questionable constriction of the left pupil to light. He scored four points for non-verbal reaction to pain (IV), a total of seven points for ability to move (V) the four extremities, and four points for reaction of both pupils (VI). His total score on day five was 15 points.

The laryngeal tube was removed the next day, and shortly thereafter the patient developed pulmonary problems with respiratory stridor and copious bronchial secretion. The graphic representation of the arterial blood gases in Fig. 1 documents the fall in $\mathrm{aPO}_{2}$ which occurred with deterioration in his neurological status. He began to recover again approximately four days later but now at a slower date of improvement of 0.48 points/day.

CASE 2

A 21 year old man (PT) fell 30 feet from a scaffold and sustained severe closed head injury. He was intubated and received supplemental oxygen. Computerised axial tomography failed to show any intracranial abnormality. On neurological examination, he was unconscious; he moved all four extremities briskly and appropriately to painful 


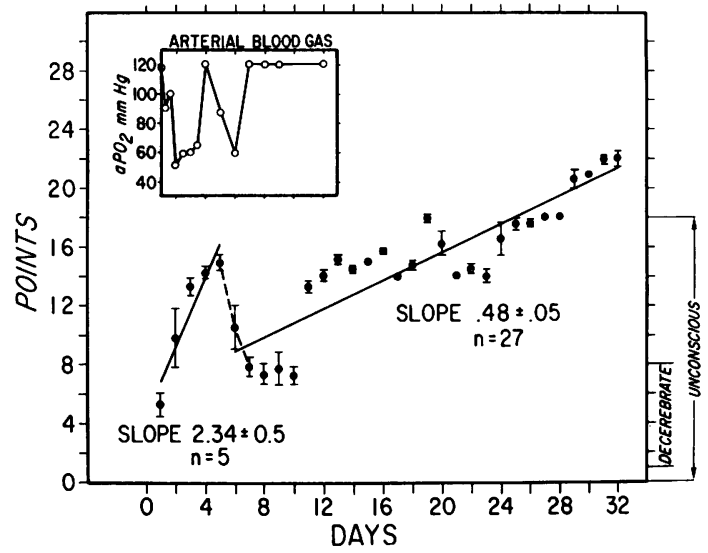

Fig. 1 Neurological score (points) determined from the Head Injury Watch Sheet (ordinate) as a function of time (days) after head injury (abscissa) in case 1. The mean values ( $\pm S E$ ) for 12-24 determinations of neurological status are plotted. The solid lines were determined by the method of least squares analysis for respective data. Insert represents arterial blood oxygen ( $\mathrm{mmHg}$ ) as a function of time (days) after initial head injury. The rate of neurological recovery (slope: points/day) was markedly altered by hypoxaemia.

stimuli, and his pupils were equal and reacted briskly to light. His scores in the subcategories of non-verbal reaction to pain (IV), ability to move (V), and reaction of pupils (VI) were four, eight, and six points respectively for a total of 18 points (Fig. 2). His HIWS scores gradually deteriorated over the next 13 days at a rate of -0.37 points/day to the range of 12-13. Repeat computerised axial tomography was performed, and was normal. Pulmonary function studies demonstrated intrapulmonary shunting and hypoxaemia unaffected by supplemental oxygen. He remained unconscious, his motor response to painful stimulus was slow but still appropriate. He then began to improve at a rate of 0.73 points/day when his respiratory difficulties were corrected two weeks after his head injury. The insert of arterial blood gas (Fig. 2) correlates his clinical status with the degree of hypoxia.

Case 2 was also scored by physicians (Fig. 3), and rate of recovery was determined to be similar and statistically comparable to the scores of the nurses (Fig. 2).

In Table 2, the rates of recovery determined by eight hour nursing shifts or by combining the three shifts into a 24 hour period are compared for case 1 . The differences in slopes were not statistically significant using Student's $t$ test. Table 3

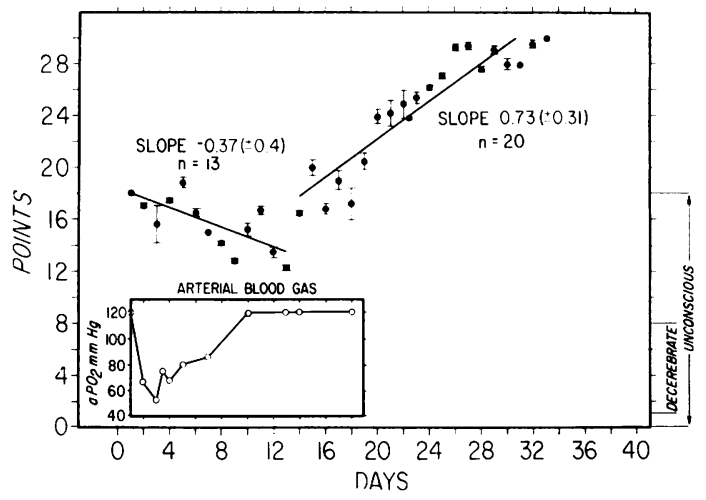

Fig. 2 Neurological score (points) determined from the Head Injury Watch Sheet (ordinate) as a function of time (days) after head injury (abscissa) in case 2.

The mean values ( $\pm S E$ ) for 12-24 determinations of neurological status daily are plotted. The slopes (points $\pm S E /$ day) are determined by the method of least squares analysis. Insert represents arterial blood oxygen $(\mathrm{mmHg})$ as a function of time after head injury. The rate of neurological deterioration as shown by negative slope occurred during the period of hypoxaemia. Neurolog:cal recovery as represented by a positive slope began after correction of hypoxaemia.

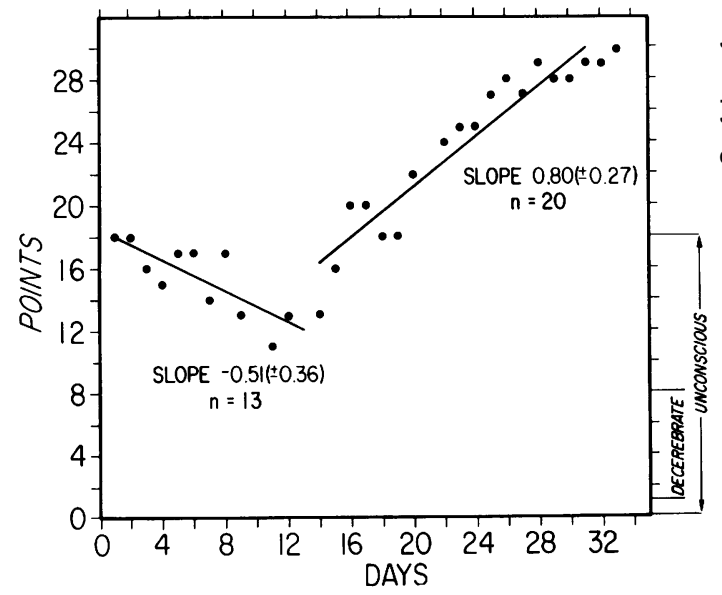

Fig. 3 Neurological score (points) determined from the Head Injury Watch Sheet (ordinate) as a function of time (days) after head injury (abscissa) in case 2.

Physicians scored patient once per day and the scores are plotted. The slopes are determined by the method of least squares analysis. The rates of deterioration and recovery are similar to those in Fig. 2.

represents the rates of recovery for case 2 and includes the comparable data determined independently by physicians. 
Table 2 Rate of recovery ( $\pm S E$ ) determined by each nursing shift ( 8 hours) and by the combination of all three shifts together (24 hours) for case 1. The rates of recovery are not significantly different using Student's $t$ test

\begin{tabular}{|c|c|c|c|c|}
\hline & \multicolumn{4}{|l|}{ Nursing shift } \\
\hline & $7-3$ & $3-11$ & $11-7$ & $24 \mathrm{hr}$ \\
\hline $\begin{array}{l}\text { 1-5 days } \\
\text { Rate of } \\
\text { recovery } \\
\text { ( } \pm \text { SE) } \\
\text { Days } \\
\text { (number) }\end{array}$ & $\begin{array}{l}2.61 \pm 0.56 \\
5\end{array}$ & $\begin{array}{l}2.48 \pm 0.65 \\
5\end{array}$ & $\begin{array}{l}1.98 \pm 0.64 \\
5\end{array}$ & $2.34 \pm 0.50$ \\
\hline $\begin{array}{l}\text { 6-32 days } \\
\text { Rate of } \\
\text { recovery } \\
\text { ( } \pm \text { SE) } \\
\text { Days } \\
\text { (number) }\end{array}$ & $\begin{array}{l}0.47 \pm 0.05 \\
27\end{array}$ & $\begin{array}{l}0.47 \pm 0.06 \\
27\end{array}$ & $\begin{array}{l}0.49 \pm 0.05 \\
27\end{array}$ & $\begin{array}{l}0.48 \pm 0.05 \\
27\end{array}$ \\
\hline
\end{tabular}

\section{Discussion}

The Head Injury Watch Sheet provides a method to quantitate the rate of recovery of a head-injured patient and is not meant to supplant the detailed neurological examination. Clinical trial showed that the HIWS is a useful and practical instrument in assessing the course of recovery after craniocerebral trauma. Nurses of varying levels of training required minimal instruction in the use of the watch sheet and readily included it in their evaluation of patients who had sustained trauma.

The accurate determination of consciousness is an important component of the HIWS. We agree with previous reports that the ability to speak is an appropriate index of consciousness (Bouzarth, 1968; Teasdale and Jennett, 1974, 1976; Jennett and Teasdale, 1977). Patients in our study, however, could not verbalise for variable lengths of time after regaining consciousness because respiratory difficulties necessitated continued intubation or tracheostomy. We found that the criteria of assessing ability of the patient to respond to command by motor movements was a practical and reliable method of assessing consciousness.
The two cases presented illustrate the sensitivity and reliability of the HIWS in detecting alteration in neurological recovery secondary to respiratory dysfunction. The score of a patient at a particular time was largely reproducible despite the involvement of different personnel at different levels of training. The rates of recovery were not significantly different whether they were scored by one nursing shift singly or by a combined average of the three shifts ( 24 hours). Furthermore, independent scoring by physicians resulted in rates of recovery similar to those determined from the nurses' examinations. This comparison further supports the reliability and validity of the method.

In conclusion, the HIWS represents a statistically reliable technique, valid in the hands of physicians and nurses, for assessing the clinical neurological status and course of the head-injured patient. The numerical assignment representing the clinical neurological assessment of a patient is drawn from a 30 point scale and may be graphically displayed. The hourly scores representing repeated determinations of the neurological status of each patient for a single day are used to determine a mean value. Daily mean scores are plotted as a function of time (days) after the initial head injury. The slopes fitted to the plotted points by the method of least squares represent the rate of change (in units per day) in the neurological status of each patient. The resulting graphic representation of a patient's clinical course facilitates the comparison of drugs or treatment regimens considered useful in patient management.

The foregoing is in no way meant to minimise the value of the Glasgow Coma Scale (Jennett and Teasdale, 1977) which attempts to quantify the level of consciousness in patients with altered responsiveness. Some problems arise, however, in the use of the Glasgow Coma Scale. The relatively limited numerical range of the scale, with three being the greatest degree of dysfunction and 15 being normal, presents a constricted scale in which minor expected examiner variation in clinical assessment

Table 3 Rate of recovery ( $\pm S E$ ) determined by each nursing shift ( 8 hours), by the combination of all three shifts together (24 hours), and by physicians for case 2.The rates of recovery are not significantly different using Student's t test

\begin{tabular}{llllll}
\hline & Nursing shift & & & \\
\cline { 2 - 5 } & $7-3$ & $3-11$ & $11-7$ & 24 hr & Physicians \\
\hline $\begin{array}{l}\text { 1-13 days } \\
\text { Rate of recovery }( \pm \text { SE) } \\
\text { Days (number) }\end{array}$ & $-0.34 \pm 0.20$ & $-0.50 \pm 0.13$ & $-0.50 \pm 0.08$ & $-0.37 \pm 0.11$ & $-0.51 \pm 0.10$ \\
$\begin{array}{l}\text { 14-33 days } \\
\text { Rate of recovery }( \pm \text { SE) } \\
\text { Days (number) }\end{array}$ & 13 & 13 & 13 & 13 & 13 \\
\hline
\end{tabular}


may have a major effect on scoring. An enlarged scale may obviate the importance of these minor fluctuations without being burdensome. Another shortcoming of the Glasgow Coma Scale is the reliance on verbal response. This is a particularly difficult area to assess early after head injury since most patients initially require endotracheal intubation. Our aim was the development of a scale useful in patients evaluation from the time of admission until achievement of self-care. This approach was felt necessary in the comparative evaluation of drugs used in patient care. The emphasis was, therefore, on determination of the rate of clinical neurological recovery rather than the prediction of future neurological status. We believe that another value of our method lies in the demonstrated validity and reliability when used by members of the nursing staff who are most common evaluators of the hour-to-hour clinical course.

This study was supported by Grant NS 13042 from the National Institute of Neurological Communicative Diseases and Stroke, National Institutes of Health. We would like to express our personal thanks to Susan Nantista and Elvira Graham for typing the manuscript.

\section{References}

Adams, F. G., and McComb, S. G. (1953). Assessment and prognosis in hemiplegia. Lancet, 1, 266-269.

Bouzarth, W. F. (1968). Neurosurgical Watch Sheet for craniocerebral trauma. Journal of Trauma, 8, 29-31.

Carlsson, C. A., Essen, C. V., and Lofgren, J. (1968). Factors affecting the clinical course of patients with severe head injuries. Journal of Neurosurgery, 29, 242-251.
Denny-Brown, D. (1950). Handbook of Neurological Examination and Case Recording. Harvard University Press: Cambridge, Massachusetts.

Jennett, B. (1976). Assessment of the severity of head injury. Journal of Neurology, Neurosurgery, and Psychiatry, 39, 647-655.

Jennett, B., and Bond, M. (1975). Assessment of outcome after severe brain damage. Lancet, 1, 480-484.

Jennett, B., and Plum, F. (1972). Persistent vegetative state after brain damage. Lancet, 1, 734-737.

Jennett, B., and Teasdale, G. (1977). Aspects of coma after severe head injury. Lancet, 1, 878-881.

Kurtzke, J. F. (1955). A new scale for evaluating disability in multiple sclerosis. Neurology (Minneapolis), 5, 580.

Lovejoy, F. H., Smith, A. L., Bresnan, M. J., Wood, J. N., Victor, D. I., and Adams, P. C. (1974). Clinical staging in Reye syndrome. American Journal of Diseases of Children, 128, 36-41.

Mathew, H., and Lawson, A. A. H. (1966). Acute barbiturate poisoning - a review of two years experience. Quarterly Journal of Medicine, 35, 539-552.

Overgaard, J., Hird-Hansen, O., Land, A., Pedersen, K. K., Christensen, S., Haase J., Hein, O., and Tweed, W. A. (1973). Prognosis after head injury based on early clinical examination. Lancet, 2, 631635.

Plum, F., and Posner, J. B. (1972). The Diagnosis of Stupor and Coma. F. A. Davis Company: Philadelphia.

Teasdale, G., and Jennett, B. (1974). Assessment of coma and impaired consciousness. Lancet, 2, 81-83.

Teasdale, G., and Jennett, B. (1976). Predicting outcome in individual patients after severe head injury. Lancet, 1, 1031-1034.

Tourtellote, W. W., Haerer, A. F., Simpson, J. R., Kuzman, J. W., and Sikorski, J. (1965). Quantificative clinical neurological testing. I. A study of a battery of tests designed to evaluate in part the neurological function of patients with multiple sclerosis and its use in a therapeutic trial. Annals of the New York Academy of Sciences, 122, 480-505. 